\title{
BER Performance Analysis of Cognitive Radio Physical Layer over Rayleigh fading Channel
}

\author{
Amandeep Kaur Virk \\ Dr. B R Ambedkar National \\ Institute of Technology, \\ Jalandhar, India
}

\author{
Ajay K Sharma \\ Dr. B R Ambedkar National \\ Institute of Technology, \\ Jalandhar, India
}

\begin{abstract}
In this paper we analyze the Bit Error Rate (BER) performance of Cognitive Radio Physical layer over Rayleigh channel under different channel encoding schemes, digital modulation schemes and channel conditions. The system outperforms with BPSK modulation technique as compared to other digital modulation schemes and the system is highly effective to combat inherent interferences under Rayleigh fading channel. The system shows improved BER on using encoding schemes with error rate reduced by $10 \%$ using Reed Solomon (RS) encoding, 95\% reduction on using Convolution encoding and 98\% error reduction on applying RS with Convolution encoding. It has been anticipated from the simulation study that the performance of the communication system degrades with the increasing of noise power.
\end{abstract}

\section{Keywords}

Cognitive Radio, Bit error rate, Additive White Gaussian Noise, Reed Solomon encoding, Convolution encoding.

\section{INTRODUCTION}

Wireless technology is embedded in our daily routine - laptops, cell phones, sensors - all are wireless devices and use a finite amount of radio spectrum. As the number of devices increase, they compete for bandwidth and the service level provided to them is degraded. Moreover, traditional fixed spectrum allocation policy can no longer meet the needs and services of the wireless users. According to Federal Communications Commission (FCC), temporal and geographical variations in the utilization of the assigned spectrum range from $15 \%$ to $85 \%$ in current spectrum allocation policies [1]. So, we need to find out ways to allow wireless devices to efficiently share the airwaves. Cognitive Radio (Dynamic Spectrum Access) has been proposed as a potential solution for spectrum inefficiency problems [2]. Cognitive Radio paradigm shifts the way the spectrum is used and this presents many challenges across various layers of cognitive radio. Cognitive Radios are quite different from traditional wireless radios, so the cognitive radio layers perform additional functionality along with functions of the conventional wireless radios.

This paper presents physical layer analysis of BER performance over a Rayleigh channel with AWGN noise. We use a Time Division Multiple Access (TDMA) system for licensed users along with Orthogonal Frequency Division Multiplexing (OFDM) and non- persistent Carrier Sense Multiple Access (CSMA) system for unlicensed users who opportunistically use the idle licensed spectrum. Various digital modulation schemes used are 8PAM, BPSK, QPSK, 8PSK, 16PSK and 16QAM. RS and Convolution encoding schemes are used with varying values of Signal to Noise ratio (SNR) to compute Bit Error Rate (BER). The paper is organized as follows: Section 2 describes the physical layer of cognitive radio, Section 3 presents the simulation results and finally, the paper is concluded in Section 4.

\section{PHYSICAL LAYER OF CR}

The physical layer in cognitive layer is responsible for:

1) Spectrum sensing

2) Reconfiguration

3) Change of operation parameters

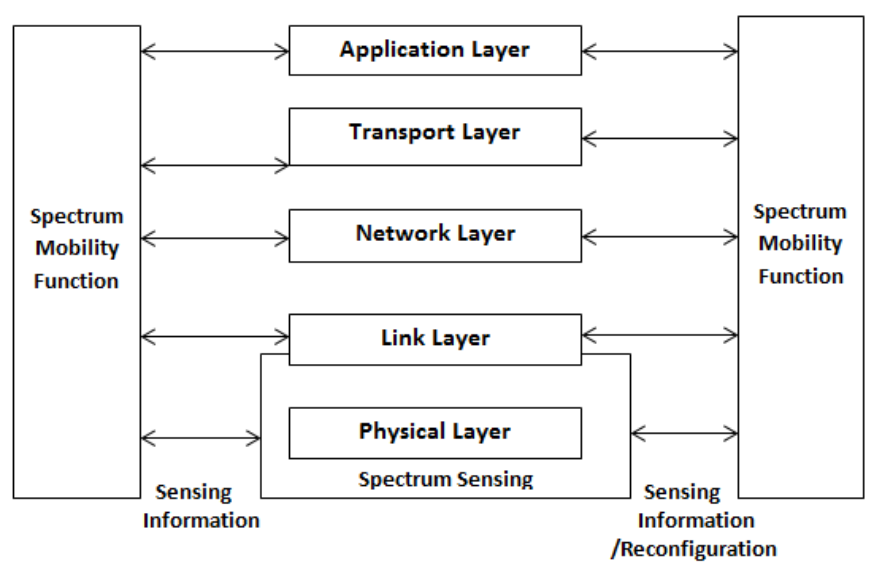

Figure 1. Cognitive Radio Layers

Spectrum sharing is detecting unused spectrum bands. Reconfiguration is done when a licensed user appears while unlicensed user is using its frequency band and operation parameters (such as frequency, power and modulation) are changed to adapt to new operating environment [3]. Figure 1 shows the various layers of cognitive radio along with physical layer functions.

Cognitive radio (CR) promises to increase spectrum usage by supporting secondary users to share licensed bands [4]. Licensed users of CR are called primary users and the unlicensed users are called secondary users. CR supports user access to the licensed spectrum as a secondary user when and where channels are detected idle [5]. 


\subsection{Access Schemes}

Multiple access schemes used in CR are: contention-based nonpersistent carrier sense multiple access (CSMA) for secondary users, and contention-free time division multiple access (TDMA) for primary users [6]. Orthogonal Frequency Division Multiplex (OFDM) is considered the ideal signal structure for wideband cognitive radio systems [7].

In an OFDM system, the data is divided into multiple parallel sub streams at a reduced data rate, and each is modulated and transmitted on a separate orthogonal subcarrier. This increases symbol duration and improves system robustness.

TDMA is used to characterize the primary user who has the license to access channel whenever they have information to send. TDMA is a contention-free multiple access scheme which usually employs a central entity (e.g. base station) to allocate capacity to individual users. It allows several users to share the same frequency channel by dividing the signal into different time slots. It provides guaranteed reserved capacity and is commonly used in packet-based communication systems, notably satellites and in the GSM standard. The strict slot reservation process makes it a suitable example of a primary user system multiple access scheme.

CSMA is used to describe the behavior of a CR secondary user that has the ability to sense and access a free channel. CSMA is a contention-based multiple access scheme where individual users make their own decisions on how and when to access a channel. The carrier frequency is sensed by each of multiple stations, or nodes, to determine network availability before accessing the medium to transmit data. Each station must monitor the network to determine if a collision has occurred and the data require retransmission. In a non- persistent CSMA, a node transmits, if the medium is idle. If the medium is busy, the node waits a random amount of time before again sensing the medium. CSMA is naturally suited to distributed networks and is currently employed in the IEEE 802.11 WLAN standard. The ability to avoid ongoing transmissions through sensing makes it a suitable example of a secondary user system multiple access scheme [8].

\section{SIMULATION RESULTS}

The modulation schemes used for simulation are 8PAM, BPSK, QPSK, 8PSK, 16PSK and16QAM. Forward error correction (FEC) encoding schemes used are convolutional encoding and RS encoding. MATLAB 7.10.0 is used for simulation. The system is observed by separately using convolutional and RS encoding and then using them in combination using inter leavers. Simulation results are shown for TDMA, CSMA and overall channel. Overall channel shows the results for TDMA and CSMA system combined together. In all the cases, the system provides degraded performance in 16 PSK and satisfactory performance in BPSK modulations. Figure 2 through 5 show the BER performance of data through CR physical layer under six types of digital modulation schemes on Rayleigh fading channel with AWGN noise.

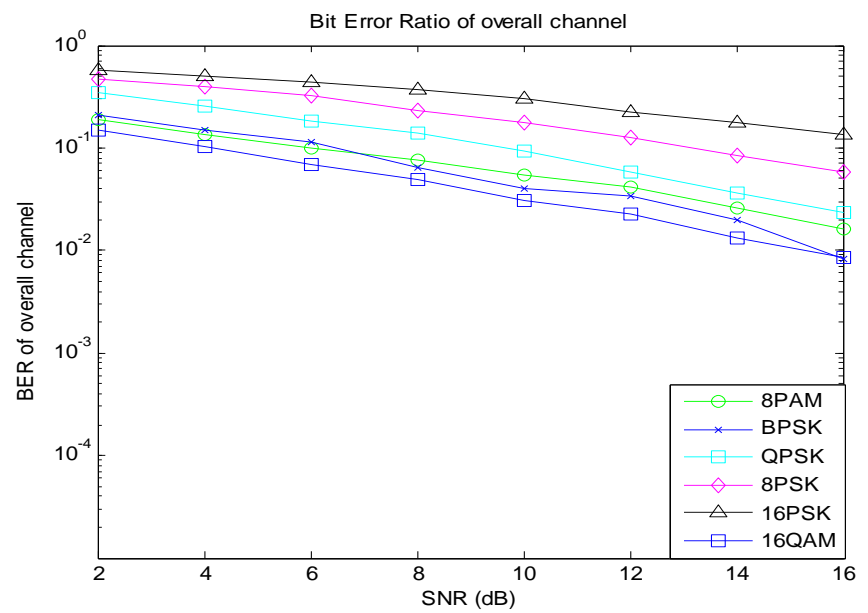

(a)

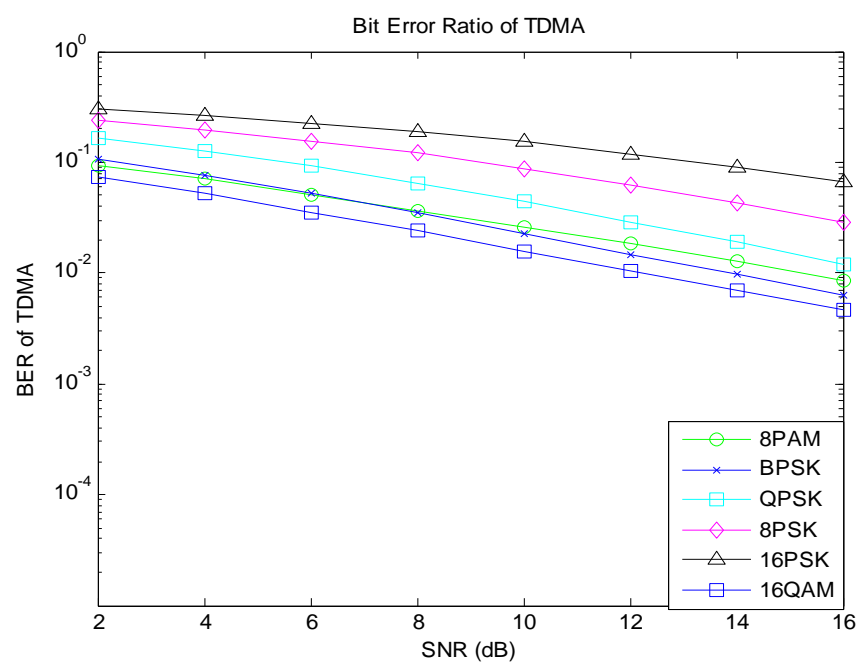

(b)

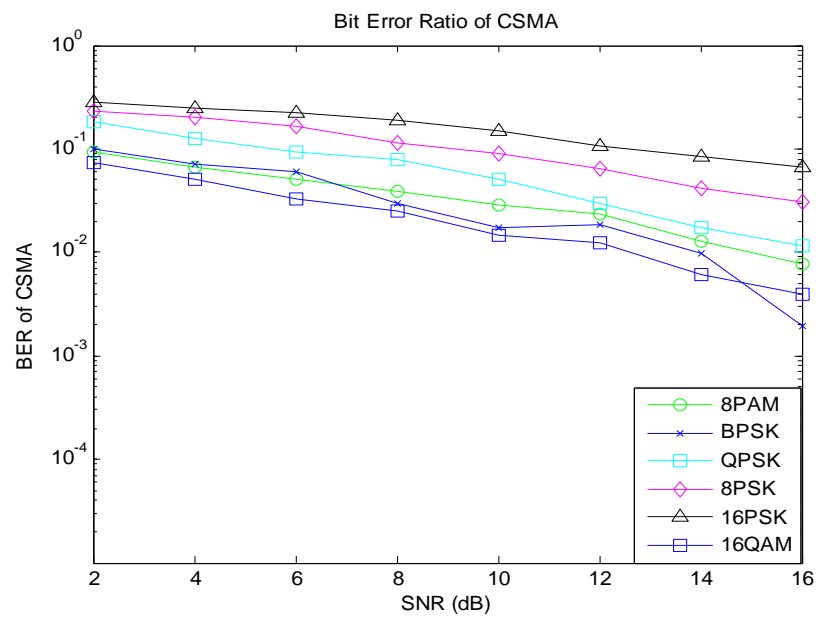

(c)

Figure2. BER simulations for CR over Rayleigh channel (a) for overall channel (b) for TDMA system (c) for CSMA system 
Figure 2 above shows the BER obtained at SNR values ranging from 2 to $16 \mathrm{~dB}$. Figure 2 (a) gives the BER of overall channel and 16QAM and BPSK perform better among all the modulation schemes with BER value $6.8755 \times 10^{-2}$ and $10.1041 \times 10^{-2}$ respectively. Figure 2 (b) gives the BER values of TDMA system. BER of 16QAM at SNR $6 \mathrm{~dB}$ is $3.5552 \times 10^{-2}$ and for BPSK is $5.091 \times 10^{-2}$ for BPSK. Figure 2 (c) shows the values of BER for a CSMA system and value of BER at SNR $6 \mathrm{~dB}$ for better performing 16QAM is $3.3203 \times 10^{-2}$ and for BPSK is $22.5466 \times 10^{-2}$.

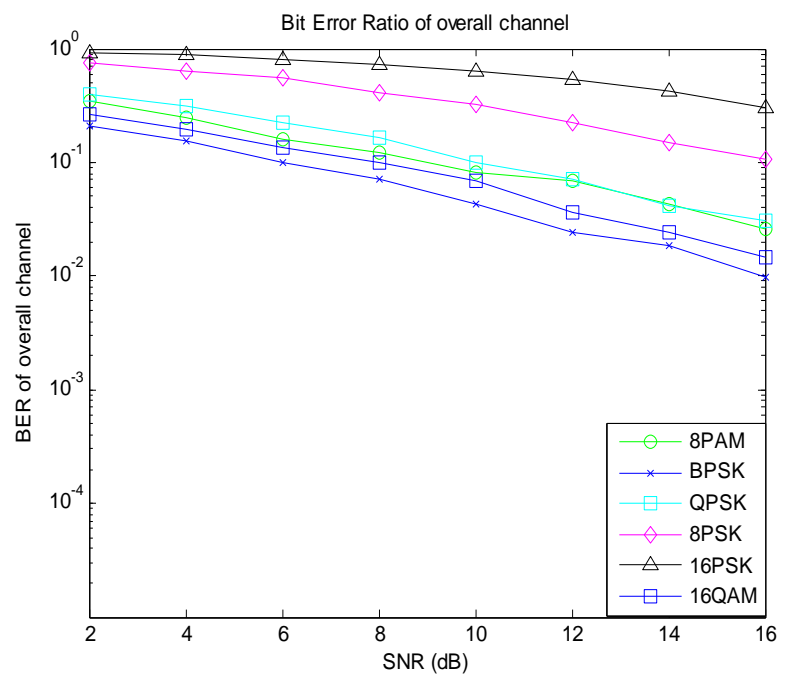

(a)

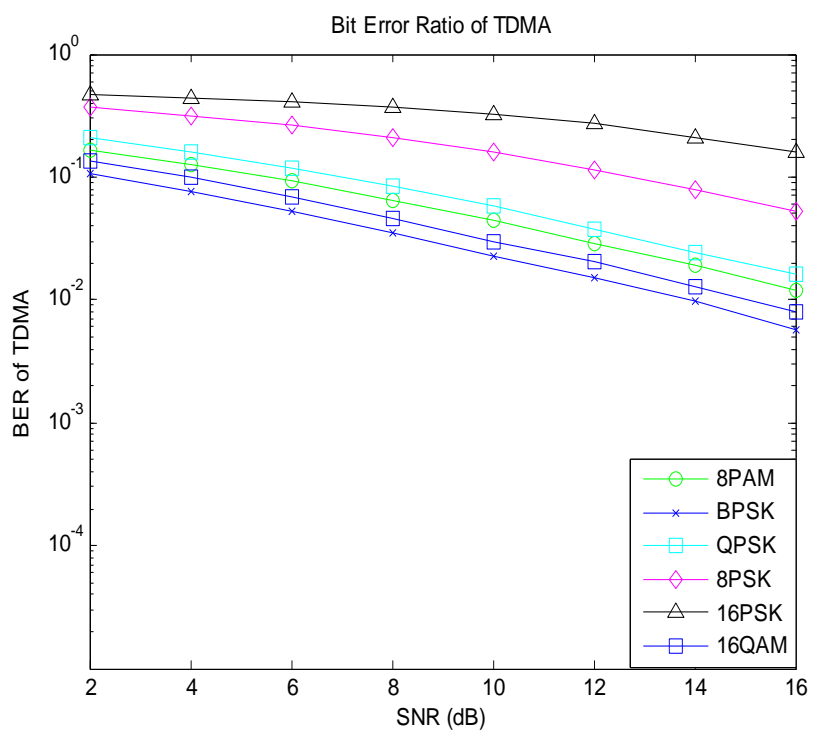

(b)

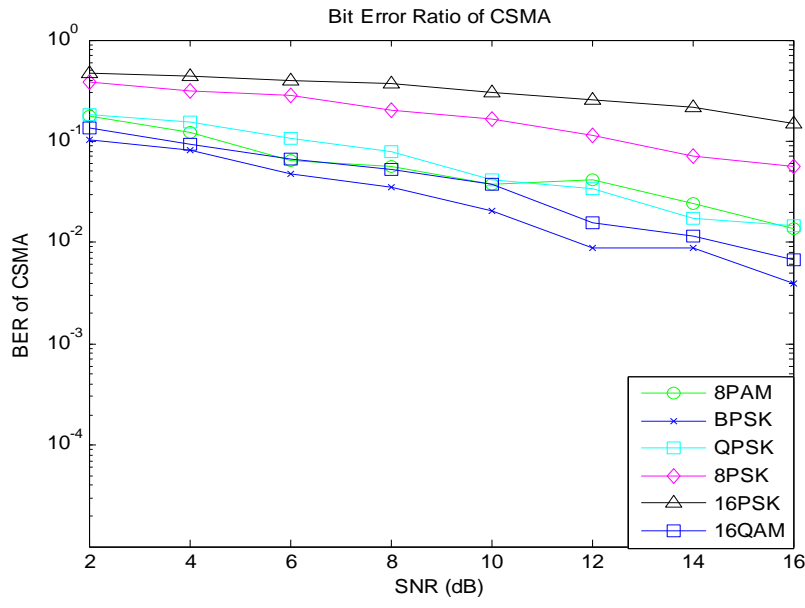

(c)

Figure 3. BER simulations for CR using RS encoded system over Rayleigh channel (a) (a) for overall channel (b) for TDMA system (c) for CSMA system

Figure 3 above shows the BER obtained at SNR values ranging from 2 to $16 \mathrm{~dB}$ for RS encoded Rayleigh channel. Figure 3 (a) gives the BER of overall channel and BPSK perform better among all the modulation schemes with BER value $9.068 \times 10^{-2}$. Figure 2 (b) gives the BER values of TDMA system. BER of BPSK at SNR $6 \mathrm{~dB}$ is $4.832 \times 10^{-2}$ for BPSK. Figure 2 (c) shows the values of BER for a CSMA system and value of BER at SNR $6 \mathrm{~dB}$ for best performing BPSK is $4.7852 \times 10^{-2}$. BPSK perform better among all modulation schemes. The system shows $10 \%$ improvement using RS encoding. BER values for BPSK over Rayleigh channel is $10.1041 \times 10^{-2}$ and improved BER using RS encoding is $9.0684 \times 10^{-2}$.

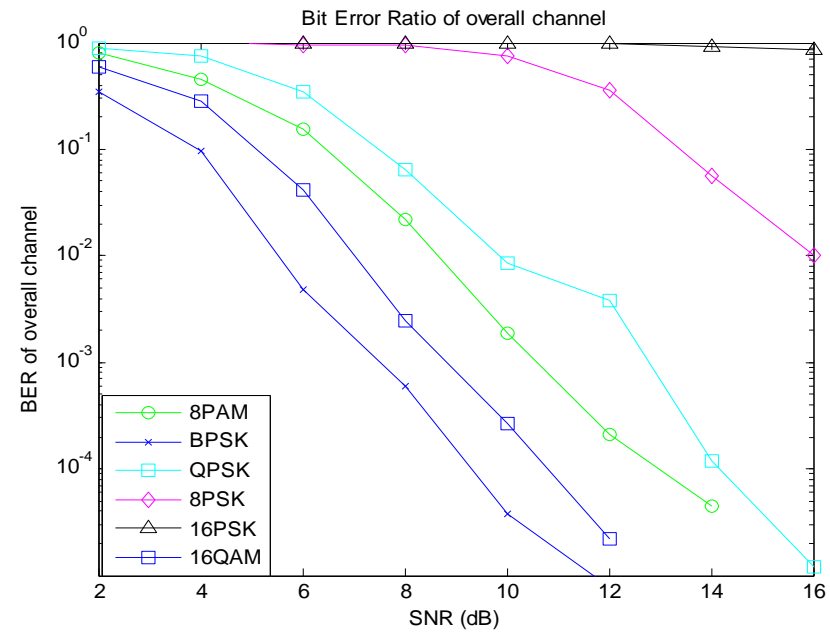

(a) 


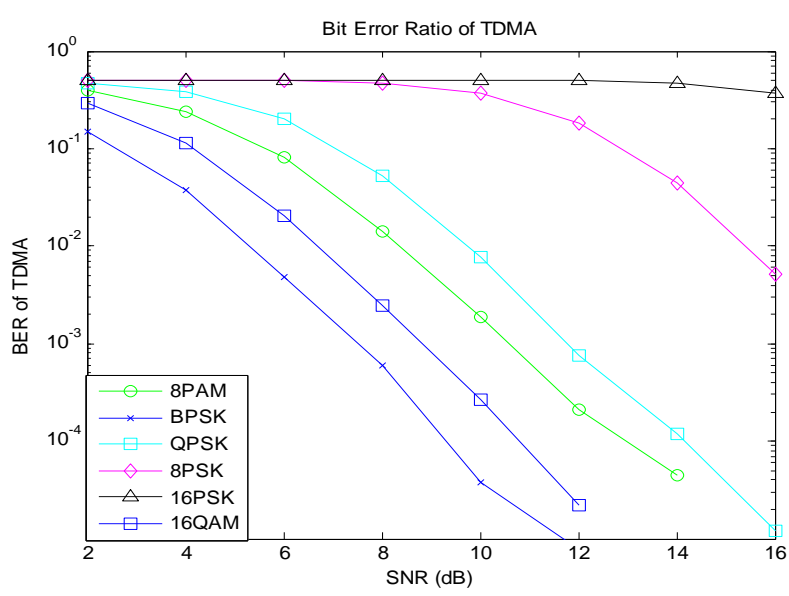

(b)

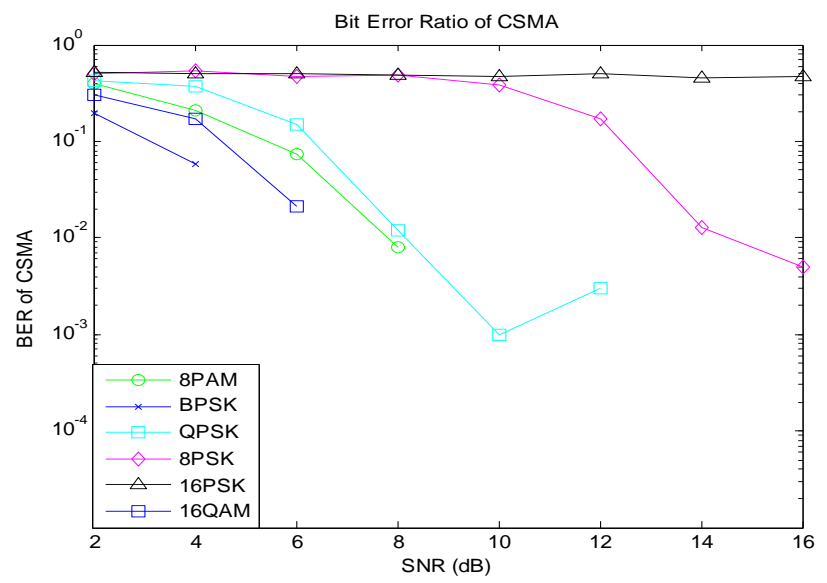

(c)

Figure 4. BER simulations for $C R$ using 1/3 convolutional encoded system over Rayleigh channel (a) for overall channel (b) for TDMA system (c) for CSMA system

The simulation results for convolution encoded Rayleigh channel are shown in figure 4 . Figure 4 (a) shows the improved results for overall channel with the improved value of BER as $4.742 \times 10^{-3}$. Improved value for TDMA system at SNR $6 \mathrm{~dB}$ is $4.742 \times 10^{-3}$ and for CSMA system is 0 as shown in figure 4(b) and (c) respectively. The BER values for BPSK modulation scheme show improvement with value for Rayleigh channel as $10.1041 \times 10^{-2}$ and for convolution encoded system as $4.742 \mathrm{x}$ $10^{-3}$. Convolution encoding removes bit errors and the system shows $95 \%$ improvement.

The simulation results for RS and convolution encoded Rayleigh channel are shown in figure 5 . Figure 5 (a), (b) and (c) show the improved results for overall channel, TDMA and CSMA system with the improved value of BER as $0.53 \times 10^{-3}, 0.537 \times 10^{-3}$ and 0 at SNR $6 \mathrm{~dB}$ respectively. RS encoding eliminates block errors and convolution encoding removes bit errors and the system shows $98 \%$ improvement.

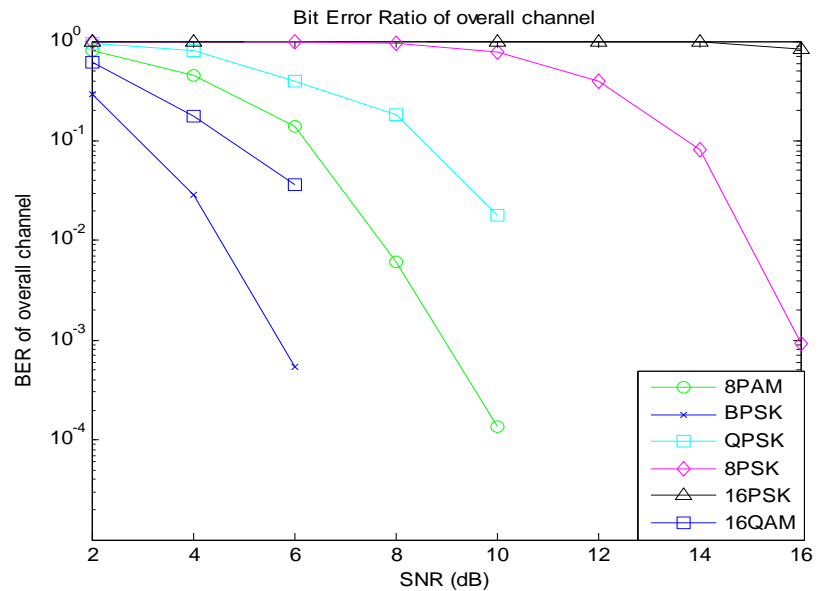

(a)

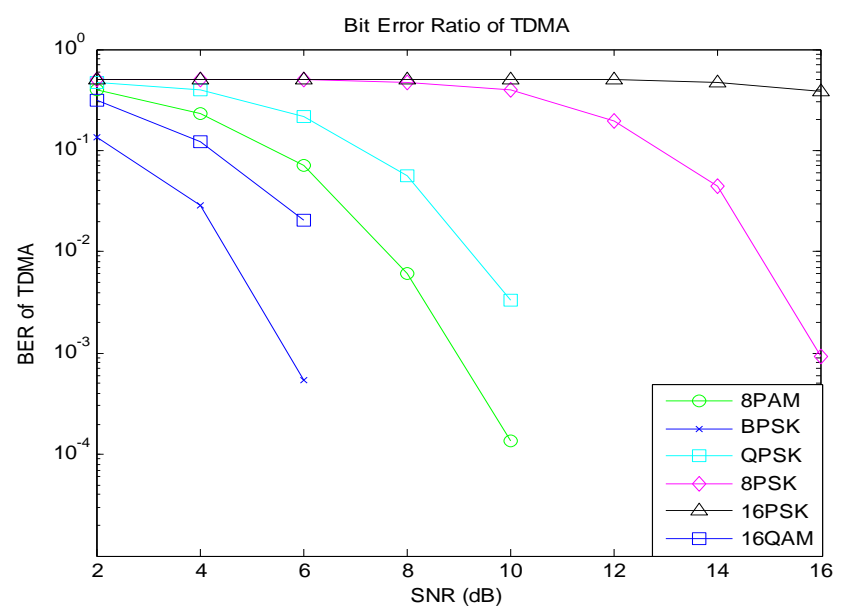

(b)

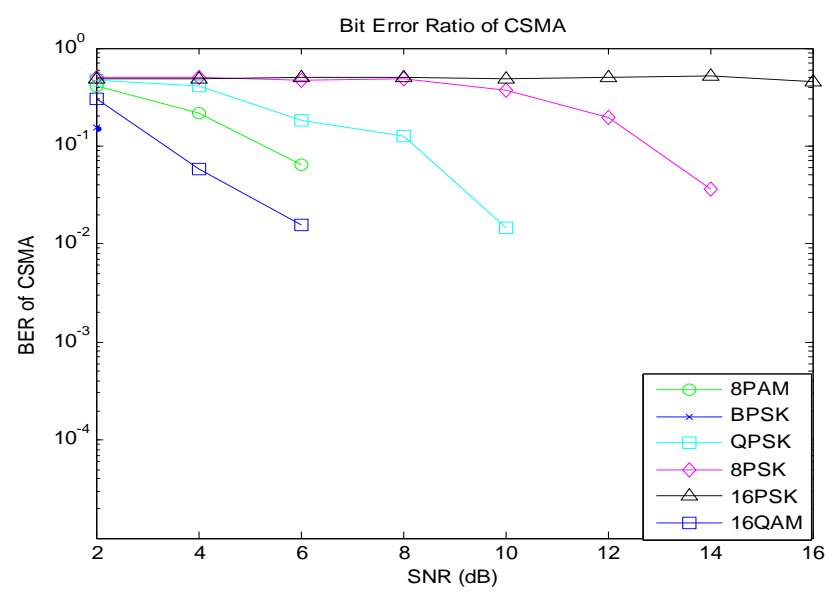

(c)

Figure 5. BER simulations for $C R$ using $R S$ and $1 / 3$ convolutional encoded system over Rayleigh channel (a) for overall channel (b) for TDMA system (c) for CSMA system

Table 1 below shows the BER values obtained using various modulation and encoding schemes. The lowest values of BER obtained are highlighted. 
Table 1. BER values for different modulation schemes for CR using Rayleigh Channel

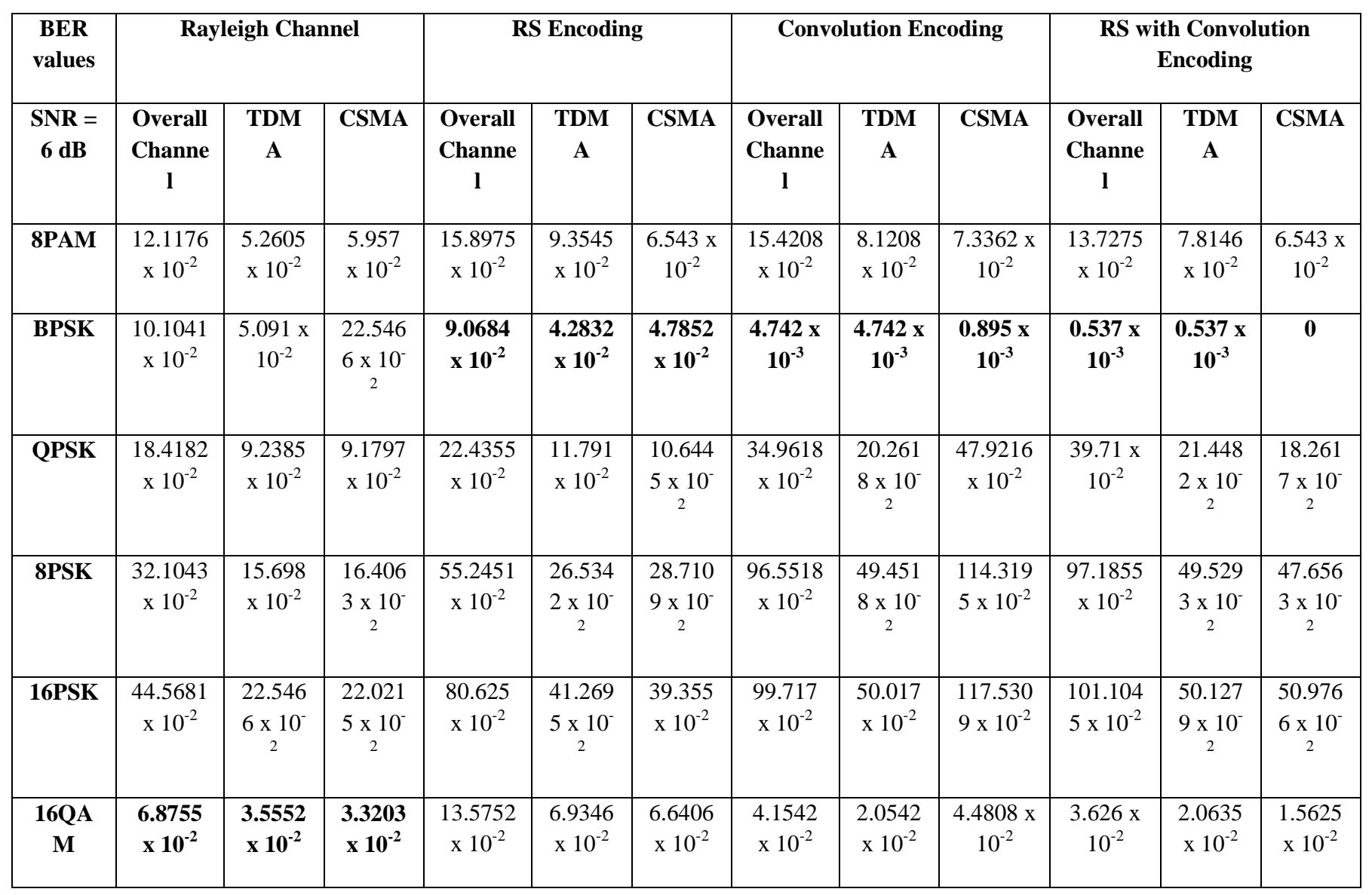

\section{CONCLUSION}

In this paper, the BER performance of data through CR physical layer is shown using RS, Convolutional channel coding and different digital modulation schemes. A range of system performance highlights the impact of digital modulations under RS and Convolution coding under Rayleigh fading channel with AWGN noise. The system shows improved performance on using encoding schemes. The enhanced BER is $10 \%$ for RS encoding, $95 \%$ for convolution encoding and $98 \%$ for RS combined with convolution encoding scheme. In the context of system performance, it can thus be concluded that the implementation of BPSK modulation with RS and convolution channel coding technique together provides satisfactory result among the digital modulation schemes with limited SNR.

\section{REFERENCES}

[1] Danijela Čabrić, Robert W. Brodersen Berkeley, "Physical Layer Design Issues Unique to Cognitive Radio Systems", Wireless Research Center, University of California at Berkeley, USA.

[2] Wang Weifang, "Denial of Service Attacks in Cognitive Radio Networks", 2nd Conference on Environmental Science and Information Application Technology,2010 IEEE.
[3] Mitola J., Maguire G.: 'Cognitive radio: making software radios more personal', IEEE Pers. Communications, 1999, 6, (4), pp. 13-18.

[4] Xueying Zhang, Cheng Li, "The security in cognitive radio networks: A survey",2009.

[5] Peha J. M.: "Approaches to spectrum sharing", IEEE Communications Mag., 2005, 43, (2), pp. 10-12.

[6] Pahlavan k., levesque a.h.: "Wireless information networks", 'Wiley Series in Telecommunications Signal.

[7] Haiyun Tang, “ Some physical layer issues of wideband cognitive radio systems", 2005, IEEE.

[8] Zhuo Yang; Yu-Dong Yao; Di Zheng,2010" TDMA for primary users and CSMA for secondary users in a cognitive radio network", Wireless and Optical Communications Conference (WOCC), 2010 19th Annual.

[9] Zhuo Yang, , "Investigations of multiple access protocols in cognitive radio networks". 2010. 